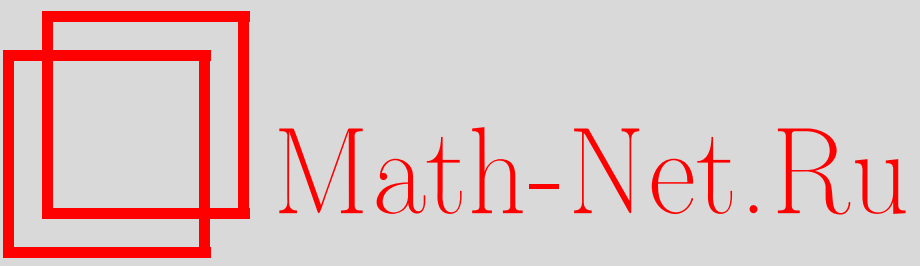

Б. М. Зупник, Частичное спонтанное нарушение трехмерной $N=2$ суперсимметрии, ТМФ, 2000, том 123, номер 1, 57-74

DOI: https://doi.org/10.4213/tmf585

Использование Общероссийского математического портала Math-Net.Ru подразумевает, что вы прочитали и согласны с пользовательским соглашением

http://www . mathnet.ru/rus/agreement

Параметры загрузки:

IP : 54.237 .59 .107

26 апреля 2023 г., 10:32:06 
ТЕОРЕТИЧЕСКАЯ

И МАТЕМАТИЧЕСКАЯ

ФИЗИКА

Том 123, № 1

апрель, 2000

(C) 2000 г.

Б. М. Зупник

\section{ЧАСТИЧНОЕ СПОНТАННОЕ НАРУШЕНИЕ ТРЕХМЕРНОЙ $N=2$ СУПЕРСИММЕТРИИ}

Обсуждаются суперполевые модели с частичным спонтанным нарушением глобальной $D=3, N=2$ суперсимметрии. Абелева калибровочная модель описывает низкоэнергетические взаимодействия вещественного скалярного поля с 3D векторным и фермионными полями. Введено новое голдстоун-максвелловское представление 3D калибровочного суперполя и доказана возможность частичного спонтанного нарушения $N=2 \rightarrow N=1$ для неминимального самодействия этого модифицированного калибровочного суперполя с включением линейного члена Файе-Илиопулоса. Рассматривается также дуальное описание частичного нарушения в модели самодействующего голдстоуновского кирального суперполя. Эти модели имеют постоянные вакуумные решения и описывают соответственно взаимодействия $N=1$ голдстоуновских мультиплетов D2-браны или супермембраны с дополнительными массивными мультиплетами.

\section{1. ВВЕДЕНИЕ}

Стандартные механизмы спонтанного нарушения глобальной $D=4, N=1$ суперсимметрии (СНГС) связаны с постоянными вакуумными решениями для вспомогательных компонент кирального и калибровочного суперполей (см. обзоры [1-3]). Постоянные СНГС-решения возможны в очень ограниченном классе взаимодействий киральных суперполей. В частности, СНГС невозможно для нетривиального самодействия одного кирального суперполя. Механизм Файе-Илиопулоса (FI) состоит в добавлении линейного члена в действие $N=1$ абелевой калибровочной теории, однако этот член не гарантирует появление СНГС-решения для любого взаимодействия калибровочных и материальных полей. Эти стандартные механизмы не достаточно гибкие, поэтому поиск новых подходов к этой проблеме весьма желателен, особенно для расширенных суперсимметрий или суперсимметрий в низших размерностях, имеюших свою специфику. Проблемы спонтанного нарушения локальных суперсимметрий не будут обсуждаться в данной работе.

* Объединенный институт ядерных исследований, Дубна, Московская обл., Россия. E-mail: zupnik@thsun1.jinr.ru 
Стандартные линейные супермультиплеты (стандартные суперполя) не удобны для описания частичного спонтанного нарушения глобальных суперсимметрий (ЧСНГС), когда инвариантность по отношению к части суперзарядов остается ненарушенной. В частности, постоянные решения с вырожденной структурой вспомогательных полей запрешены во многих случаях. Модели голдстоуновских фермионов с частичным спонтанным нарушением $D=4, N=2[4]$ или $D=3, N=2$ [5] суперсимметрий были построены с использованием топологически нетривиальных классических решений, сохраняющих половину суперзарядов. Эти модели также изучались в методе нелинейных реализаций суперсимметрий [6-11], использующем суперполя ненарушенной суперсимметрии.

Абелева калибровочная модель с двумя FI-членами была использована для спонтанного нарушения $D=4, N=2$ суперсимметрии до ее $N=1$ подгруппы [12-14]. Эта модель описывает неминимальные взаимодействия комплексного скалярного поля с фермионными и $U(1)$-калибровочным полями. В $D=4, N=2$ суперпространстве эти взаимодействия соответствуют голоморфному действию голдстоун-максвелловского (ГМ) кирального суперполя $W$, удовлетворяющего модифицированным суперполевым связям второго порядка. По сравнению с исходными связями $N=2$ векторного мультиплета [15] эти связи содержат постоянные члены, которые гарантируют появление постоянных мнимых частей вспомогательной изовекторной компоненты и голдстоуновской фермионной компоненты в ГМ-суперполе.

Ранее пример связи голдстоуновского типа был рассмотрен для модели с частичным спонтанным нарушением $D=1, N=4$ суперсимметрии в [16]. Таким образом, эти связи вводят новый тип представлений суперсимметрии с линейными голдстоуновскими (ЛГ) фермионами. В отличие от голдстоуновских фермионов нелинейных реализаций, преобразующихся линейно только в ненарушенной суперсимметрии, ЛГ-фермионы имеют партнеров в супермультиплетах полной суперсимметрии. Нелинейные деформации стандартных связей также возможны [3], однако мы будем обсуждать только постоянные члены в модифицированных связях, которые имеют отношение к спонтанному нарушению суперсимметрий. Будет показано, что использование ЛГ-векторного мультиплета и соответствуюшего дуального скалярного мультиплета решает проблему частичного спонтанного нарушения $D=3, N=2$ суперсимметрии. Эта проблема недавно рассматривалась в рамках $N=1$ суперпространства [11].

Координаты полного $D=3, N=2$ суперпространства

$$
z=\left(x^{\alpha \beta}, \theta^{\alpha}, \bar{\theta}^{\alpha}\right)
$$

имеют спинорные индексы $\alpha, \beta$ группы $S L(2, R)$. Спинорное представление $x$-координаты связано с векторным представлением через $3 \mathrm{D} \gamma$-матрицы $x^{\alpha \beta}=(1 / 2) x^{m}\left(\gamma_{m}\right)^{\alpha \beta}$. Алгебра спинорных производных в этом суперпространстве имеет следующий вид:

$$
\begin{aligned}
& \left\{\mathcal{D}_{\alpha}, \overline{\mathcal{D}}_{\beta}\right\}=i \partial_{\alpha \beta}+i \varepsilon_{\alpha \beta} Z, \\
& \left\{\mathcal{D}_{\alpha}, \mathcal{D}_{\beta}\right\}=0, \quad\left\{\overline{\mathcal{D}}_{\alpha}, \overline{\mathcal{D}}_{\beta}\right\}=0,
\end{aligned}
$$


где $Z$ - вешественный центральный заряд,

$$
\begin{array}{ll}
\mathcal{D}_{\alpha}=D_{\alpha}+\frac{i}{2} \bar{\theta}_{\alpha} Z, & D_{\alpha}=\partial_{\alpha}+\frac{i}{2} \bar{\theta}^{\beta} \partial_{\alpha \beta}, \\
\overline{\mathcal{D}}_{\alpha}=\bar{D}_{\alpha}-\frac{i}{2} \theta_{\alpha} Z, & \bar{D}_{\alpha}=\bar{\partial}_{\alpha}+\frac{i}{2} \theta^{\beta} \partial_{\alpha \beta} .
\end{array}
$$

Мы будем использовать главным образом спинорные производные без центрального заряда $D_{\alpha}$ и $\bar{D}_{\alpha}$.

Соответствующие генераторы $N=2$ суперсимметрии имеют вид

$$
\mathcal{Q}_{\alpha}=Q_{\alpha}+\frac{1}{2} \bar{\theta}_{\alpha} Z, \quad \overline{\mathcal{Q}}_{\alpha}=\bar{Q}_{\alpha}-\frac{1}{2} \theta_{\alpha} Z
$$

Алгебра $N=2$ суперсимметрии ковариантна относительно $U_{R}(1)$-преобразований

$$
\theta^{\alpha} \rightarrow e^{i \rho} \theta^{\alpha}, \quad \bar{\theta}^{\alpha} \rightarrow e^{-i \rho} \bar{\theta}^{\alpha}
$$

Мы будем рассматривать следующие обозначения для билинейных комбинаций спинорных координат и дифференциальных операторов:

$$
\begin{aligned}
(\theta)^{2} & =\frac{1}{2} \theta_{\alpha} \theta^{\alpha}, & (\bar{\theta})^{2} & =\frac{1}{2} \bar{\theta}^{\alpha} \bar{\theta}_{\alpha}, \\
(\theta \bar{\theta}) & =\frac{1}{2} \theta^{\alpha} \bar{\theta}_{\alpha}, & \Theta^{\alpha \beta} & =\frac{1}{2}\left[\theta^{\alpha} \bar{\theta}^{\beta}+\alpha \leftrightarrow \beta\right], \\
(D)^{2} & =\frac{1}{2} D^{\alpha} D_{\alpha}, & (\bar{D})^{2} & =\frac{1}{2} \bar{D}_{\alpha} \bar{D}^{\alpha}, \\
(D \bar{D}) & =\frac{1}{2} D^{\alpha} \bar{D}_{\alpha}, & D_{\alpha \beta} & =\frac{1}{2}\left(\left[D_{\alpha}, \bar{D}_{\beta}\right]+\alpha \leftrightarrow \beta\right),
\end{aligned}
$$

а также учитывать полезные соотношения

$$
\begin{aligned}
D_{\alpha} \bar{D}_{\beta} & =\frac{i}{2} \partial_{\alpha \beta}+\varepsilon_{\alpha \beta}(D \bar{D})+\frac{1}{2} D_{\alpha \beta}, \\
(D \bar{D})^{2} & =\frac{1}{8} \partial^{\alpha \beta}\left(\partial_{\alpha \beta}-i D_{\alpha \beta}\right)+\frac{1}{2}(D)^{2}(\bar{D})^{2}, \\
D_{\alpha \beta}(D)^{2} & =i \partial_{\alpha \beta}(D)^{2}, \\
(D)^{2}(\theta)^{2} & =1, \quad(\bar{D})^{2}(\bar{\theta})^{2}=1, \quad(D \bar{D})(\theta \bar{\theta})=-\frac{1}{2} .
\end{aligned}
$$

В полном и киральном суперпространствах используются меры интегрирования

$$
d^{7} z=d^{3} x(D)^{2}(\bar{D})^{2}, \quad d^{5} \zeta=d^{3} x_{L}(D)^{2}
$$

которые имеют $R$-заряды 0 и -2 , соответственно. Комплексные киральные координаты строятся по аналогии со случаем $D=4$ :

$$
\zeta=\left(x_{L}^{\alpha \beta}, \theta^{\alpha}\right), \quad x_{L}^{\alpha \beta}=x^{\alpha \beta}+i \Theta^{\alpha \beta} .
$$


Удобно использовать следуюшие правила сопряжения для любых операторов [3]:

$$
(X Y)^{\dagger}=Y^{\dagger} X^{\dagger}, \quad[X, Y\}^{\dagger}=-(-1)^{p(X) p(Y)}\left[X^{\dagger}, Y^{\dagger}\right\}
$$

где $[X, Y\}$ - градуированный коммутатор, $p(X)= \pm 1-Z_{2}$-четность. Действие дифференциального оператора $X$ на некоторую функцию $f(z)$ и соответствуюшее сопряжение определяются как

$$
X f \equiv[X, f\} \Rightarrow(X f)^{\dagger}=-(-1)^{p(X) p(f)} X^{\dagger} f^{\dagger}
$$

(Отметим, что также возможно альтернативное определение сопряжения $(X f)^{\dagger}=$ $\left.(-1)^{p(X) p(f)} X^{\dagger} f^{\dagger} \cdot\right)$

Рассмотрим правила сопряжения для спинорных координат и производных

$$
\begin{aligned}
& \left(\theta^{\alpha}\right)^{\dagger}=\bar{\theta}^{\alpha}, \quad\left[(\theta)^{2}\right]^{\dagger}=(\bar{\theta})^{2}, \quad(\theta \bar{\theta})^{\dagger}=-(\theta \bar{\theta}), \\
& D_{\alpha}^{\dagger}=\bar{D}_{\alpha}, \quad\left[(D)^{2}\right]^{\dagger}=(\bar{D})^{2}, \quad(D \bar{D})^{\dagger}=-(D \bar{D}) .
\end{aligned}
$$

Можно ввести вешественные $N=2$ спинорные координаты $\theta_{i}^{\alpha}=\left(\theta_{i}^{\alpha}\right)^{\dagger}$,

$$
\begin{array}{rlrl}
\theta^{\alpha} & =\frac{1}{\sqrt{2}}\left(\theta_{1}^{\alpha}+i \theta_{2}^{\alpha}\right), & \bar{\theta}^{\alpha}=\frac{1}{\sqrt{2}}\left(\theta_{1}^{\alpha}-i \theta_{2}^{\alpha}\right), \\
(\theta)^{2} & =\frac{1}{2}\left[\left(\theta_{2} \theta_{2}\right)-\left(\theta_{1} \theta_{1}\right)-2 i\left(\theta_{1} \theta_{2}\right)\right], & & \left(\theta_{i} \theta_{k}\right) \equiv \frac{1}{2} \theta_{i}^{\alpha} \theta_{k \alpha}=\left(\theta_{k} \theta_{i}\right), \\
(\theta \bar{\theta}) & =\frac{1}{2}\left[\left(\theta_{1} \theta_{1}\right)+\left(\theta_{2} \theta_{2}\right)\right], & & \left(\theta_{i} \theta_{k}\right)^{\dagger}=-\left(\theta_{i} \theta_{k}\right), \\
\Theta^{\alpha \beta} & =\frac{i}{2}\left(\theta_{2}^{\alpha} \theta_{1}^{\beta}+\alpha \leftrightarrow \beta\right), & \left(\Theta^{\alpha \beta}\right)^{\dagger}=\Theta^{\alpha \beta},
\end{array}
$$

и соответствуюшие спинорные производные

$$
\begin{aligned}
& \mathcal{D}_{\alpha}^{1}=D_{\alpha}^{1}+\frac{1}{2} \theta_{2 \alpha} Z, \quad \mathcal{D}_{\alpha}^{2}=D_{\alpha}^{2}-\frac{1}{2} \theta_{1 \alpha} Z \\
& D_{\alpha}^{1}=\frac{1}{\sqrt{2}}\left(D_{\alpha}+\bar{D}_{\alpha}\right), \quad D_{\alpha}^{2}=\frac{i}{\sqrt{2}}\left(D_{\alpha}-\bar{D}_{\alpha}\right), \\
& \left\{D_{\alpha}^{1}, D_{\beta}^{1}\right\}=\left\{D_{\alpha}^{2}, D_{\beta}^{2}\right\}=i \partial_{\alpha \beta}, \quad\left\{D_{\alpha}^{1}, D_{\beta}^{2}\right\}=0 .
\end{aligned}
$$

$D=3, N=2$ калибровочные теории рассматривались, например, в работах [17-20]. Неминимальное самодействие $U(1)$-калибровочного супермультиплета в этом случае эквивалентно взаимодействию 3D линейного мультиплета. Мы будем анализировать модифицированные ЛГ (мЛГ)-связи для $3 \mathrm{D}$ калибровочного мультиплета. Соответствующее вещественное $N=2$ суперполе описьвает голдстоуновские фермионы, взаимодействующие со скалярным и векторным полями. 
В разделе 4 мы обсуждаем препотенциальное решение для мЛГ-супермультиплета, которое содержит дополнительные члены, явно зависящие от спинорных координат и некоторых комплексных констант, играющих роль модулей в вакуумном состоянии теории совместно с константой FI-члена. Используя это представление в неминимальном калибровочном действии, можно получить постоянные вакуумные решения с частичным нарушением $D=3, N=2$ суперсимметрии. Отметим, что алгебра суперсимметрии модифицируется на мЛГ-препотенциале $V$ по аналогии с подобными модифицированными преобразованиями 4D калибровочных полей или препотенциалов $[13,14]$.

Раздел 5 посвящен описанию ЧСНГС во взаимодействии ЛГ-кирального суперполя, которое дуально взаимодействию мЛГ-суперполя. Это явно суперсимметричное действие зависит от суммы кирального и антикирального суперполей, а также некоторого постоянного члена, билинейного по спинорным координатам. Необычное преобразование основного ЛГ-кирального суперполя удовлетворяет алгебре суперсимметрии с центральным зарядом.

Действия $N=1$ супермембраны и D2-браны [11] могут быть проанализированы в нашем подходе с использованием разложения $N=2$ суперполей по второй спинорной координате $\theta_{2}^{\alpha}$. В разделе 6 рассматриваются $N=1$ компоненты расширенных суперполей и ковариантные условия, которые позволяют нам выразить дополнительные степени свободы через голдстоуновские суперполя.

\section{2. ВЕКТОРНЫЙ МУЛЬТИПЛЕТ В $D=3, \quad N=2$ СУПЕРСИММЕТРИИ}

$D=3, N=2$ калибровочная теория [17-20] аналогична хорошо известной $D=4$, $N=1$ калибровочной теории, хотя трехмерный случай имеет некоторые интересные особенности, которые связаны с существованием топологического массового члена и дуальностью между $3 \mathrm{D}$ векторным и киральным мультиплетами. Мы будем рассматривать базисное суперпространство с $Z=0$.

Абелев $U(1)$-калибровочный препотенциал $V(z)$ имеет калибровочное преобразование

$$
\delta V=\Lambda+\bar{\Lambda}
$$

где рассматриваются киральный и антикиральный параметры

$$
\bar{D}_{\alpha} \Lambda=0, \quad D_{\alpha} \bar{\Lambda}=0
$$

$D=3, N=2$ векторный мультиплет описывается вешественным линейным суперполем

$$
W(V)=i(D \bar{D}) V
$$

удовлетворяюшим связям

$$
(D)^{2} W=(\bar{D})^{2} W=0 \text {. }
$$


Дополнительные полезные соотношения для этого суперполя имеют следуюший вид:

$$
\begin{aligned}
D_{\alpha}(D \bar{D}) W & =-\frac{i}{2} \partial_{\alpha \beta} D^{\beta} W, \\
(D \bar{D})^{2} W & =\frac{1}{8} \partial^{\alpha \beta}\left(\partial_{\alpha \beta}-i D_{\alpha \beta}\right) W .
\end{aligned}
$$

Компоненты векторного мультиплета вычисляются как не зависяшие от $\theta$ части основных суперполей и их спинорных производных:

$$
\begin{aligned}
\varphi(x) & =\left.W\right|_{0}=\left.i(D \bar{D}) V\right|_{0}, & \lambda_{\alpha}(x) & =\left.D_{\alpha} W\right|_{0}, \\
\bar{\lambda}_{\alpha}(x) & =-\left.\bar{D}_{\alpha} W\right|_{0}, & A_{\alpha \beta}(x) & =\left.D_{\alpha \beta} V\right|_{0}, \\
F_{\alpha \beta}(x) & =\left.D_{\alpha \beta} W\right|_{0}, & G(x) & =\left.i(D \bar{D}) W\right|_{0},
\end{aligned}
$$

где $A_{\alpha \beta}$ и $F_{\alpha \beta}-3 \mathrm{D}$ векторное поле и его напряженность, $G$ - вешественная вспомогательная компонента, $\varphi, \lambda$ и $\bar{\lambda}$ - физические скалярное и спинорные поля. Скалярное поле появляется как $3 \mathrm{D}$ аналог третьей компоненты $4 \mathrm{D}$ калибровочного поля.

Низкоэнергетическое эффективное действие $3 \mathrm{D}$ векторного мультиплета описывает неминимальное взаимодействие вешественного скалярного поля с фермионным и калибровочным полями. Для $U(1)$-калибровочного суперполя $V$ это действие имеет следующий вид:

$$
S(W)=-\frac{1}{2} \int d^{7} z H(W), \quad \tau(W)=H^{\prime \prime}(W)>0,
$$

где $H(W)$ - вешественная выпуклая функция $W$. Отметим, что действие $S(W)$ сохраняет $U_{R}(1)$-инвариантность.

Интересная особенность $3 \mathrm{D}$ калибровочной теории связана с существованием члена Черна-Саймонса [17]

$$
S_{\mathrm{CS}}=\frac{i k}{4} \int d^{7} z V(D \bar{D}) V
$$

где $k$ - некоторая константа. Компонентная форма этого действия содержит топологический калибровочный член

$$
\int d^{3} x A_{\alpha \beta} \partial_{\gamma}^{\alpha} A^{\gamma \beta}
$$

Заметим, что неабелево обобщение этого члена построено в работе [18].

$3 \mathrm{D}$ линейньй мультиплет дуален киральному мультиплету $\phi$. Преобразование Лежандра, описываюшее эту дуальность,

$$
S[B, \Phi]=-\frac{1}{2} \int d^{7} z[H(B)-\Phi B]
$$

включает вешественное неограниченное суперполе $B$ и суперполе $\Phi=\phi+\bar{\phi}$. Варьируя множители Лагранжа $\phi$ и $\bar{\phi}$, можно получить связи (2.4). 
Используя решение алгебраического $B$-уравнения

$$
H^{\prime}(B) \equiv f(B)=\Phi
$$

можно перейти к самодействию киральных суперполей:

$$
\begin{aligned}
& B \Rightarrow B(\Phi)=f^{-1}(\Phi), \\
& S(\Phi)=-\frac{1}{2} \int d^{7} z \widehat{H}(\Phi), \\
& \widehat{H}(\Phi)=H[B(\Phi)]-\Phi B(\Phi), \\
& \frac{\partial \Phi}{\partial B}=\tau(B), \quad \frac{\partial^{2} \widehat{H}}{\partial \Phi^{2}} \equiv \hat{\tau}=-\frac{1}{\tau} .
\end{aligned}
$$

Соответствующее суперполевое уравнение имеет вид

$$
(\bar{D})^{2} \widehat{H}^{\prime}(\Phi)=\hat{\tau}(\Phi)(\bar{D})^{2} \bar{\phi}+\frac{1}{2} \hat{\tau}^{\prime}(\Phi) \bar{D}_{\alpha} \bar{\phi} \bar{D}^{\alpha} \bar{\phi}=0
$$

Эта киральная модель описывает специальный случай кэлеровой суперсимметричной $\sigma$-модели, которая полностью определяется вещественной функцией $H$ и обладает по построению дополнительной абелевой изометрией

$$
\phi \rightarrow \phi+i \beta
$$

где $\beta$ - некоторый вещественньй параметр.

\section{3. ТРУДНОСТИ СО СПОНТАННЫМ НАРУШЕНИЕМ СУПЕРСИММЕТРИИ}

Рассмотрим спонтанное нарушение суперсимметрии в неминимальной калибровочной модели (2.10) с добавочным линейным FI-членом

$$
S_{\mathrm{FI}}=\frac{1}{2} \xi \int d^{7} z V,
$$

где $\xi$ - константа размерности - 1 . Варьируя суперполе $V$, можно вывести соответствующее суперполевое уравнение движения

$$
-i(D \bar{D}) H^{\prime}(W)+\xi=-i \tau(W)(D \bar{D}) W-\frac{i}{2} \tau^{\prime}(W) D^{\alpha} W \bar{D}_{\alpha} W+\xi=0
$$

где

$$
\tau(W)=H^{\prime \prime}(W), \quad \tau^{\prime}(W)=H^{\prime \prime \prime}(W)
$$


Спинорные производные этого уравнения порождают компонентные уравнения движения различной размерности

$$
\begin{aligned}
D_{\alpha}(D \bar{D}) H^{\prime}= & -\frac{i}{2} \tau(W) \partial_{\alpha \beta} D^{\beta} W+\frac{1}{2} \tau^{\prime}(W) D_{\alpha} W(D \bar{D}) W+ \\
& +\frac{1}{2} \tau^{\prime}(W) D^{\beta} W\left(D_{\alpha \beta}+\frac{i}{2} \partial_{\alpha \beta}\right) W- \\
& -\frac{1}{4} \tau^{\prime \prime}(W) \bar{D}_{\alpha} W D^{\beta} W D_{\beta} W-\frac{1}{2} \tau^{\prime}(W) \bar{D}_{\alpha} W(D)^{2} W=0
\end{aligned}
$$

где последний член исчезает вследствие условия (2.4). Вакуумные решения можно анализировать с помощью уравнения

$$
(D \bar{D})^{2} H^{\prime}(W)=0
$$

Мы будем изучать постоянные решения уравнений движения, используя следующую вакуумную подстановку:

$$
V_{0}=2 i(\theta \bar{\theta}) a-2(\theta)^{2}(\bar{\theta})^{2} G, \quad W_{0}=a+2 i(\theta \bar{\theta}) G
$$

где $a$ и $G$ - константы. Низшие вакуумные компоненты уравнений (3.2) и (3.5) имеют вид

$$
\begin{gathered}
G \tau(a)-\xi=0, \\
G^{2} \tau^{\prime}(a)=0 .
\end{gathered}
$$

Нетривиальное решение $G_{0} \neq 0$ возможно только для квадратичной функции $H$.

Полезно рассматривать вещественные $3 \mathrm{D}$ спиноры $\lambda_{i}^{\alpha}=\left(\lambda_{i}^{\alpha}\right)^{\dagger}$,

$$
\lambda^{\alpha}=\frac{1}{\sqrt{2}}\left(\lambda_{1}^{\alpha}+i \lambda_{2}^{\alpha}\right)
$$

и соответствуюшие вешественные спинорные параметры $N=2$ суперсимметрии

$$
\epsilon^{\alpha}=\frac{1}{\sqrt{2}}\left(\epsilon_{1}^{\alpha}+i \epsilon_{2}^{\alpha}\right)
$$

Ясно, что постоянное решение вида (3.6) может нарушать спонтанно только обе суперсимметрии

$$
\delta_{\epsilon} \lambda^{\alpha}=i G_{0} \bar{\epsilon}^{\alpha}, \quad \delta_{\epsilon} \bar{\lambda}^{\alpha}=-i G_{0} \epsilon^{\alpha}
$$

так как оно порождает два вещественных голдстоуновских фермиона. 
Таким образом, полное спонтанное нарушение $N=2$ суперсимметрии возможно только для $W^{2}(V)$-взаимодействия с FI-членом. Частичное спонтанное нарушение запрещено, если использовать векторный мультиплет, удовлетворяющий стандартному условию (2.4).

Оценим роль члена Черна-Саймонса (2.11) в вакуумных уравнениях. Варьируя действие $S(W)+S_{\mathrm{CS}}+S_{\mathrm{FI}}$, можно получить модифицированное уравнение движения

$$
-i(D \bar{D}) H^{\prime}+k W+\xi=0
$$

Это суперполевое уравнение дает следуюшие модифицированные вакуумные уравнения:

$$
\begin{aligned}
& G \tau(a)-k a-\xi=0, \\
& G^{2} \tau^{\prime}(a)-k G=0 .
\end{aligned}
$$

Скалярный потенциал этой модели имеет вид

$$
\mathcal{V}_{k}(a)=\frac{1}{2 \tau(a)}(\xi+k a)^{2} .
$$

Для произвольной функции $\infty>\tau(a)>0$ этот потенциал имеет единственный явно суперсимметричный минимум $a=-\xi k^{-1}$. Таким образом, даже свободное СНГС-решение $\tau=$ const исчезает в присутствии члена Черна-Саймонса.

\section{4. ЧАСТИЧНОЕ СПОНТАННОЕ НАРУШЕНИЕ СУПЕРСИММЕТРИИ}

Мы определим модифицированные связи голдстоуновского типа для 3D векторного мультиплета аналогично тому, как это было сделано в работах $[16,14]$, и покажем, что спонтанное нарушение $D=3, N=2$ суперсимметрии возможно для нетривиального калибровочного взаимодействия в этом подходе.

Рассмотрим следующую деформацию связей (2.4):

$$
(D)^{2} \widehat{W}=C, \quad(\bar{D})^{2} \widehat{W}=\bar{C},
$$

где $C$ и $\bar{C}$ - некоторые константы. Эти соотношения явно нарушают $U_{R}(1)$-инвариантность.

Решение этих связей можно построить по аналогии с (2.3):

$$
\widehat{W}=i(D \bar{D}) V+(\theta)^{2} C+(\bar{\theta})^{2} \bar{C} .
$$

Это мЛГ-суперполе содержит новые постоянные вспомогательные структуры, которые радикально меняют матрицу вакуумных фермионных преобразований:

$$
\begin{aligned}
& \delta_{\epsilon} \lambda^{\alpha}=-C \epsilon^{\alpha}+i G_{0} \bar{\epsilon}^{\alpha}, \\
& \delta_{\epsilon} \bar{\lambda}^{\alpha}=-i G_{0} \epsilon^{\alpha}-\bar{C} \bar{\epsilon}^{\alpha} .
\end{aligned}
$$

3 Теоретическая и математическая физика, т. 123, № 1, 2000 г. 
Очевидно, что ЧСНГС-условие соответствует вырожденности этих преобразований:

$$
C \bar{C}-G_{0}^{2}=0
$$

В этом случае можно выбрать одно вешественное голдстоуновское спинорное поле как линейную комбинацию $\lambda_{i}^{\alpha}$. Для случая чисто мнимой константы $C$ ЛГ-фермион можно отождествить с $\lambda_{2}^{\alpha}$.

Следует подчеркнуть, что сдвинутая величина $W(V)=i(D \bar{D}) V(4.2)$ не является стандартным суперполем:

$$
\delta_{\epsilon} \widehat{W}=i \epsilon_{k}^{\alpha} Q_{\alpha}^{k} \widehat{W} \rightarrow \delta_{\epsilon} W(V)=C \epsilon^{\alpha} \theta_{\alpha}-\bar{C} \bar{\epsilon}^{\alpha} \bar{\theta}_{\alpha}+i \epsilon_{k}^{\alpha} Q_{\alpha}^{k} W(V) .
$$

Алгебра этих преобразований не меняется на калибровочно-инвариантном суперполе:

$$
\left[\delta_{\eta}, \delta_{\epsilon}\right] W(V)=\epsilon_{k}^{\alpha} \eta_{l}^{\beta}\left\{Q_{\alpha}^{k}, Q_{\beta}^{l}\right\} W(V)
$$

Преобразование мЛГ-препотенциала $V$ будет рассмотрено в конце этого раздела.

Действие мЛГ-суперполя (4.2) имеет вид

$$
\widehat{S}(V)=-\frac{1}{2} \int d^{7} z[H(\widehat{W})-\xi V]
$$

и зависит от трех констант $\xi, C$ и $\bar{C}$.

Члены без производных в компонентном лагранжиане

$$
\frac{1}{2}\left(G^{2}-|C|^{2}\right) \tau(\varphi)-\xi G
$$

дают следуюший скалярный потенциал:

$$
\mathcal{V}(\varphi)=\frac{1}{2}\left[|C|^{2} \tau(\varphi)+\xi^{2} \tau^{-1}(\varphi)\right]
$$

Вакуумные уравнения в этой модели имеют вид

$$
\begin{aligned}
& G \tau(a)-\xi=0, \\
& \left(G^{2}-|C|^{2}\right) \tau^{\prime}(a)=0 .
\end{aligned}
$$

ЧСНГС-решение (4.5) возникает для нетривиального взаимодействия $\tau^{\prime}(a) \neq 0$. Это решение определяет точку минимума $a_{0}$ модели:

$$
\tau\left(a_{0}\right)=\frac{|\xi|}{|C|} .
$$


Вакуумное вспомогательное поле можно вычислить в точке $a_{0}$ :

$$
G_{0}=\frac{\xi}{\tau\left(a_{0}\right)}= \pm|C|
$$

Используя $U_{R}(1)$-преобразование, можно выбрать чисто мнимую константу $C \rightarrow c=$ $i|c|$ (без ограничения общности), тогда

$$
G_{0}=-i c=|c|
$$

Этот выбор соответствует следуюшему разложению мЛГ-суперполя (4.2):

$$
\begin{aligned}
\widehat{W} & =W_{s}\left(V_{s}\right)+2 i|c|\left(\theta_{2} \theta_{2}\right), \\
W_{s}\left(V_{s}\right) & =\frac{i}{4}\left(D^{1 \alpha} D_{\alpha}^{1}+D^{2 \alpha} D_{\alpha}^{2}\right) V_{s},
\end{aligned}
$$

где $V_{s}$ - сдвинутый мЛГ-препотенциал, который имеет исчезающее вакуумное решение для вспомогательной компоненты. Очевидно, что это представление нарушает спонтанно только $N=2$ суперсимметрию.

Рассмотрим преобразования суперсимметрии $W_{s}$ и $V_{s}$ :

$$
\begin{aligned}
\delta_{\epsilon} W_{s} & =i \epsilon_{k}^{\alpha} Q_{\alpha}^{k} \widehat{W}=-2 i|c| \epsilon_{2}^{\alpha} \theta_{2 \alpha}+i \epsilon_{k}^{\alpha} Q_{\alpha}^{k} W_{s} \\
\delta_{\epsilon} V_{s} & =\Delta(\epsilon, \theta)+i \epsilon_{k}^{\alpha} Q_{\alpha}^{k} V_{s} \\
\Delta(\epsilon, \theta) & =2|c| \epsilon_{2}^{\alpha} \theta_{2 \alpha}\left(\theta_{1}^{\beta} \theta_{1 \beta}\right)=-2 \sqrt{2}|c| i \epsilon_{2}^{\alpha}\left[\bar{\theta}_{\alpha}(\theta)^{2}+\theta_{\alpha}(\bar{\theta})^{2}\right] .
\end{aligned}
$$

Алгебра суперсимметрии $V_{s}$-преобразований существенно модифицируется по аналогии с преобразованиями препотенциалов в работах $[13,14]$ :

$$
\begin{aligned}
{\left[\delta_{\eta}, \delta_{\epsilon}\right] V_{s} } & \equiv \epsilon_{k}^{\alpha} \eta_{l}^{\beta}\left\{\widetilde{Q}_{\alpha}^{k}, \widetilde{Q}_{\beta}^{l}\right\} V_{s}= \\
& =4|c|\left(\epsilon_{2}^{\alpha} \eta_{1}^{\beta}-\eta_{2}^{\alpha} \epsilon_{1}^{\beta}\right) \theta_{1 \beta} \theta_{2 \alpha}+\epsilon_{k}^{\alpha} \eta_{l}^{\beta}\left\{Q_{\alpha}^{k}, Q_{\beta}^{l}\right\} V_{s},
\end{aligned}
$$

где $\widetilde{Q}_{\alpha}^{k}$ - генераторы модифицированных преобразований.

Измененная часть алгебры преобразований суперсимметрии имеет следующий вид:

$$
\left\{\widetilde{Q}_{\alpha}^{1}, \widetilde{Q}_{\beta}^{2}\right\}_{\bmod } V_{s}=4|c| \theta_{1 \alpha} \theta_{2 \beta}=4 i|c| \Theta_{\alpha \beta}+2 i|c| \varepsilon_{\alpha \beta}\left[(\theta)^{2}+(\bar{\theta})^{2}\right]
$$

Следует подчеркнуть, что оба члена в этом антикоммутаторе могут быть представлены в виде суммы киральных и антикиральных функций и не дают вклада в скобку Ли на суперполе $W_{s}$ :

$$
\Theta^{\alpha \beta}=-\frac{i}{2}\left(x_{L}^{\alpha \beta}-x_{R}^{\alpha \beta}\right), \quad x_{R}^{\alpha \beta} \equiv\left(x_{L}^{\alpha \beta}\right)^{\dagger} .
$$


Модифищированный антикоммутатор содержит дополнительные векторные и скалярные генераторы $T_{\alpha \beta}, T$ и $\bar{T}$ :

$$
\begin{aligned}
& \left\{\widetilde{Q}_{\alpha}^{1}, \widetilde{Q}_{\beta}^{2}\right\}=\varepsilon_{\alpha \beta}(T+\bar{T})+T_{\alpha \beta}, \\
& T_{\alpha \beta} V_{s}=4 i|c| \Theta_{\alpha \beta}, \quad T V_{s}=2 i|c|(\theta)^{2} .
\end{aligned}
$$

Дополнительные генераторы принадлежат к бесконечной алгебре Ли $U(1)$-калибровочных преобразований, которая возникает в $(x, \theta)$-разложении киральных калибровочных параметров $\Lambda$ (2.1). Эти генераторы исчезают на калибровочно-инвариантной величине $W_{s}$. Следует также включить в модифицированную алгебру $N=2$ суперсимметрии все нетривиальные коммутаторы $T$ генераторов со спинорными генераторами $\widetilde{Q}_{\alpha}^{k}$.

Рассмотрим спинорную калибровочную связность

$$
A_{\alpha}(z)=D_{\alpha} V_{s}, \quad \delta_{\Lambda} A_{\alpha}=D_{\alpha} \Lambda
$$

в киральном представлении $\left(\bar{A}_{\alpha}=0\right)$. Неоднородный член в модифицированном преобразовании суперсимметрии этого калибровочного суперполя имеет следующий вид:

$$
\delta A_{\alpha}=-2 \sqrt{2} i|c|\left[\epsilon_{2 \alpha}(\bar{\theta})^{2}-\theta_{\alpha} \epsilon_{2}^{\beta} \bar{\theta}_{\beta}\right]+i \epsilon_{k}^{\alpha} Q_{\alpha}^{k} A_{\alpha}
$$

Следует заметить, что минимальное взаимодействие заряженных киральных суперполей с мЛГ-препотенциалом $V_{s}$ нарушает суперсимметрию. Аналогичная проблема мЛГ-взаимодействия с заряженной материей появляется также в ЧСНГС-модели с $D=4, N=2$ суперсимметрией [14].

\section{3D КИРАЛЬНОЕ ВЗАИМОДЕЙСТВИЕ С ЧАСТИЧНЫМ НАРУШЕНИЕМ}

Общее эффективное действие киральных суперполей $\phi_{i} \quad(i-$ некоторый внутренний индекс) может быть записано в следующем виде:

$$
\int d^{4} x d^{4} \theta K\left(\phi_{k}, \bar{\phi}_{k}\right)+\left[\int d^{4} x d^{2} \theta P\left(\phi_{i}\right)+\text { c.c. }\right]
$$

где $K$ - кэлеров потенциал, $P$ - киральный суперполевой потенциал.

Сушествование СНГС-решения возможно в случае вырожденности матрицы $\partial_{i} \partial_{k} P$. Вакуумное уравнение для одного кирального суперполя $\phi$ может иметь неисчезающее СНГС-решение только в тривиальном случае линейной функции $P(\phi)$ и свободного кэлерова потенциала $K=\phi \bar{\phi}$. 
Мы покажем, что спонтанное нарушение суперсимметрии возможно для нетривиального взаимодействия ЛГ-кирального суперполя, которое обладает неоднородным преобразованием суперсимметрии. Рассмотрим дуальную картину для калибровочной ЧСНГС-модели с FI-членом (4.8)

$$
\widehat{S}(B, \phi, \bar{\phi})=-\frac{1}{2} \int d^{7} z[H(B)-B \widehat{\Phi}]-\frac{1}{2}\left[\bar{C} \int d^{5} \zeta \phi+\text { c. c. }\right],
$$

где введено модифицированное ограниченное ЛГ-суперполе

$$
\widehat{\Phi} \equiv \phi+\bar{\phi}+2 i \xi(\theta \bar{\theta}), \quad(D \bar{D}) \widehat{\Phi}=-i \xi
$$

Варьируя киральный и антикиральный множители Лагранжа $\phi$ и $\bar{\phi}$, можно получить мЛГ-связи (4.2) на суперполе $B$ и затем перейти к калибровочной фазе $B \rightarrow \widehat{W}(V)$, где член $(\theta \bar{\theta})$ в $\widehat{\Phi}$ преобразуется в FІ-член.

Алгебраическое $B$-уравнение

$$
H^{\prime}(B) \equiv f(B)=\widehat{\Phi}
$$

порождает переход к киральной фазе

$$
B \rightarrow f^{-1}(\widehat{\Phi}) \equiv \widehat{B}(\widehat{\Phi})
$$

Преобразованное киральное действие имеет вид

$$
\begin{aligned}
& \widehat{S}(\widehat{\Phi})=-\frac{1}{2} \int d^{7} z\left\{\widehat{H}(\widehat{\Phi})+\left[\bar{C}(\theta)^{2}+\text { c. c. }\right] \widehat{\Phi}\right\}, \\
& \widehat{H}(\widehat{\Phi})=H[\widehat{B}(\widehat{\Phi})]-\widehat{\Phi} \widehat{B}(\widehat{\Phi}) .
\end{aligned}
$$

Линейные члены, содержашие $C$ и $\bar{C}$, нарушают $U_{R}(1)$-симметрию (1.6), однако это действие инвариантно по отношению к преобразованию изометрии (2.19).

Следует подчеркнуть, что ЛГ-суперполе $\widehat{\Phi}$ преобразуется однородно, тогда как преобразование суперсимметрии ЛГ-кирального множителя Лагранжа $\phi$ содержит неоднородный член

$$
\delta_{\epsilon} \phi=-i \xi\left(\theta^{\alpha} \bar{\epsilon}_{\alpha}\right)+i \epsilon_{k}^{\alpha} Q_{\alpha}^{k} \phi
$$

Действие $\widehat{S}$ инвариантно относительно ЛГ-представления $N=2$ суперсимметрии, так как первый член этого действия явно зависит от ковариантного суперполя $\widehat{\Phi}$, а второй член инвариантен из-за линейной $\theta$-зависимости неоднородной части $\delta_{\epsilon} \phi$.

Рассмотрим $\theta$-разложение ЛГ-кирального суперполя

$$
\phi=A\left(x_{L}\right)+\theta^{\alpha} \psi_{\alpha}\left(x_{L}\right)+(\theta)^{2} F\left(x_{L}\right),
$$

где $x_{L}-$ комплексная координата кирального базиса. 
Скобка Ли модифицированных преобразований суперсимметрии (5.8)

$$
\left[\delta_{\eta}, \delta_{\epsilon}\right] \phi=i \xi\left(\epsilon^{\alpha} \bar{\eta}_{\alpha}-\eta^{\alpha} \bar{\epsilon}_{\alpha}\right)+\epsilon_{k}^{\alpha} \eta_{l}^{\beta}\left\{Q_{\alpha}^{k}, Q_{\beta}^{l}\right\} \phi
$$

содержит составной параметр центрального заряда, соответствующий следуюшему действию генератора $Z$ на киральном суперполе:

$$
Z \phi=\xi, \quad Z \bar{\phi}=-\xi .
$$

Таким образом, в этой модели появляется голдстоуновское бозонное поле $\operatorname{Im} A(x)$ для преобразований центрального заряда. Следует заметить, что преобразование изометрии (2.19) в киральной модели без ЧСНГС нельзя отождествить с преобразованием центрального заряда.

Интересно, что мы можем определить деформированное киральное суперполе

$$
\begin{gathered}
\phi_{\xi}=\phi+i \xi(\theta \bar{\theta})=e^{i(\theta \bar{\theta}) Z} \phi, \quad Z^{2} \phi=0, \\
\overline{\mathcal{D}}_{\alpha} \phi_{\xi}=\left(\bar{D}_{\alpha}-\frac{i}{2} \theta_{\alpha} Z\right) \phi_{\xi}=0,
\end{gathered}
$$

удовлетворяюшее необычному ковариантному условию.

Суперполевое уравнение движения для действия (5.6)

$$
(\bar{D})^{2} \widehat{H}^{\prime}(\widehat{\Phi})+\bar{C}=0
$$

порождает вакуумные компонентные уравнения

$$
\begin{aligned}
& \bar{F} \hat{\tau}(b)+\bar{C}=0, \quad b=A+\bar{A}, \\
& \left(|F|^{2}-\xi^{2}\right) \hat{\tau}^{\prime}(b)=0, \\
& \hat{\tau}=\widehat{H}^{\prime \prime}=-\tau^{-1} .
\end{aligned}
$$

Скалярньй потенциал этой модели зависит только от одной вешественной скалярной компоненты:

$$
\mathcal{V}(b)=\frac{1}{2}\left[\xi^{2} \hat{\tau}(b)+|C|^{2} \hat{\tau}^{-1}(b)\right] .
$$

Точка минимума $b_{0}$ этого потенциала определяется уравнением

$$
\begin{gathered}
\mathcal{V}^{\prime}=\frac{1}{2} \hat{\tau}^{\prime}(b)\left[\xi^{2}-|C|^{2} \hat{\tau}^{-2}(b)\right]=0, \\
\tau^{2}\left(b_{0}\right)=\hat{\tau}^{-2}=\xi^{2}|C|^{2}
\end{gathered}
$$

с использованием условия $\tau^{\prime}(b) \neq 0$.

Вакуумные преобразования спинорных компонент ЛГ-суперполей $\phi$ и $\bar{\phi}$ имеют следующий вид:

$$
\begin{aligned}
& \delta_{\epsilon} \psi^{\alpha}=F_{0} \epsilon^{\alpha}-i \xi \bar{\epsilon}^{\alpha}, \\
& \delta_{\epsilon} \bar{\psi}^{\alpha}=i \xi \epsilon^{\alpha}+\bar{F}_{0} \bar{\epsilon}^{\alpha} .
\end{aligned}
$$

Вакуумное решение $\left|F_{0}\right|^{2}=\xi^{2}$ соответствует условию вырожденности для этих преобразований. Выбор $F_{0}=i \xi$ нарушает $N=2$ суперсимметрию.

Таким образом, нетривиальное взаимодействие ЛГ-кирального суперполя $\phi$ приводит к частичному спонтанному нарушению $D=3, N=2$ суперсимметрии. Это явление также анализировалось в формализме $D=3, N=1$ голдстоуновских суперполей [11]. 


\section{6. ПЕРЕХОД К $N=1$ СУПЕРПОЛЯМ}

Предположим, что спинорные координаты $\theta_{1}^{\alpha}$ параметризуют $N=1$ суперпространство, а генераторы $Q_{\alpha}^{1}$ образуют соответствуюшую подалгебру $N=2$ суперсимметрии. Комплексные киральные координаты $\zeta$ (1.16) могут быть выражены через вешественные спинорные координаты

$$
\begin{aligned}
x_{L}^{\alpha \beta} & =x^{\alpha \beta}+\frac{1}{2}\left(\theta_{1}^{\alpha} \theta_{2}^{\beta}+\alpha \leftrightarrow \beta\right), \\
\theta^{\alpha} & =\frac{1}{\sqrt{2}}\left(\theta_{1}^{\alpha}+i \theta_{2}^{\alpha}\right) .
\end{aligned}
$$

Мы будем использовать соотношения

$$
\begin{aligned}
& (D)^{2}=\frac{1}{2}\left(D^{1} D^{1}\right)-\frac{1}{2}\left(D^{2} D^{2}\right)-i\left(D^{1} D^{2}\right), \quad\left(D^{i} D^{k}\right) \equiv \frac{1}{2} D^{i \alpha} D_{\alpha}^{k}, \\
& D_{\alpha}^{1} D_{\beta}^{1}=\frac{i}{2} \partial_{\alpha \beta}+\varepsilon_{\alpha \beta}\left(D^{1} D^{1}\right), \quad\left\{D_{\alpha}^{1},\left(D^{1} D^{1}\right)\right\}=0, \\
& {\left[D_{\alpha}^{1},\left(D^{1} D^{1}\right)\right]=-i \partial_{\alpha \beta} D^{1 \beta}, \quad\left(D^{1} D^{1}\right)^{2}=\frac{1}{8} \square_{3}, \quad \square_{3}=\partial^{\alpha \beta} \partial_{\alpha \beta} .}
\end{aligned}
$$

Условие киральности в вешественном базисе

$$
\bar{D}_{\alpha} \phi=\frac{1}{\sqrt{2}}\left(D_{\alpha}^{1}+i D_{\alpha}^{2}\right) \phi=0
$$

может быть решено с использованием неограниченного комплексного $N=1$ суперполя $\chi$ :

$$
\phi=\chi\left(x, \theta_{1}\right)+i \theta_{2}^{\alpha} D_{\alpha}^{1} \chi\left(x, \theta_{1}\right)+\left(\theta_{2} \theta_{2}\right)\left(D^{1} D^{1}\right) \chi\left(x, \theta_{1}\right) .
$$

Для доказательства киральности в $N=1$ представлении следует использовать уравнения $(6.4),(6.5)$ и соотношение

$$
D_{\alpha}^{2} \chi\left(x, \theta_{1}\right)=\frac{i}{2} \theta_{2}^{\beta} \partial_{\alpha \beta} \chi\left(x, \theta_{1}\right)
$$

Используя (6.3), легко получить соотношение между киральным и $N=1$ интегралами

$$
\int d^{3} x(D)^{2} \phi=\int d^{3} x\left(D^{1} D^{1}\right) \chi\left(x, \theta_{1}\right),
$$

где $d^{2} \theta_{1}=\left(D^{1} D^{1}\right)$ - мнимая спинорная мера $N=1$ суперпространства.

Преобразование (5.8) имеет $N=1$ разложение

$$
\delta \phi=-\frac{1}{2} \xi \theta_{1}^{\alpha}\left(\epsilon_{2 \alpha}+i \epsilon_{1 \alpha}\right)+\frac{1}{2} \xi \theta_{2}^{\alpha}\left(\epsilon_{1 \alpha}-i \epsilon_{2 \alpha}\right)+\epsilon_{2}^{\alpha}\left(-\partial_{\alpha}^{2}+\frac{i}{2} \theta_{2}^{\beta} \partial_{\alpha \beta}\right) \phi+i \epsilon_{1}^{\alpha} Q_{\alpha}^{1} \phi
$$


и порождает следуюшее преобразование комплексного $N=1$ суперполя:

$$
\delta \chi=-\frac{1}{2} \xi \theta_{1}^{\alpha}\left(\epsilon_{2 \alpha}+i \epsilon_{1 \alpha}\right)-i \epsilon_{2}^{\alpha} D_{\alpha}^{1} \chi+i \epsilon_{1}^{\alpha} Q_{\alpha}^{1} \chi
$$

Рассмотрим $\theta_{2}$-разложение основного суперполя (5.3) киральной ЧСНГС-модели

$$
\begin{aligned}
& \widehat{\Phi}=\chi+\bar{\chi}+i \theta_{2}^{\alpha} D_{\alpha}^{1}(\chi-\bar{\chi})+\left(\theta_{2} \theta_{2}\right)\left(D^{1} D^{1}\right)(\chi+\bar{\chi})+i \xi\left[\left(\theta_{1} \theta_{1}\right)+\left(\theta_{2} \theta_{2}\right)\right]= \\
& \quad=\Sigma+\theta_{2}^{\alpha} D_{\alpha}^{1} \rho+\left(\theta_{2} \theta_{2}\right)\left[\left(D^{1} D^{1}\right) \Sigma+2 i \xi\right] \\
& \quad \Sigma\left(x, \theta_{1}\right)=\chi+\bar{\chi}+i \xi\left(\theta_{1} \theta_{1}\right), \quad \rho=i \chi-i \bar{\chi}
\end{aligned}
$$

где $\Sigma$ - массивное вешественное $N=1$ суперполе, $\rho$ - вешественное голдстоуновское суперполе для $N=2$ суперсимметрии:

$$
\begin{aligned}
\delta \Sigma & =-\epsilon_{2}^{\alpha} D_{\alpha}^{1} \rho+i \epsilon_{1}^{\alpha} Q_{\alpha}^{1} \Sigma, \\
\delta \rho & =-i \xi \epsilon_{2}^{\alpha} \theta_{1 \alpha}+\epsilon_{2}^{\alpha} D_{\alpha}^{1} \Sigma+i \epsilon_{1}^{\alpha} Q_{\alpha}^{1} \rho .
\end{aligned}
$$

Аналогичные преобразования $N=1$ суперполей предложены в работе [11], где показано, что дополнительное суперполе может быть выражено через спинорную производную голдстоуновского суперполя $\rho$ с целью построения действия супермембраны. Массивные степени свободы в этом подходе могут быть устранены с помощью ковариантного условия

$$
\widehat{\Phi}^{2}=0
$$

которое позволяет выразить $\Sigma$ через $D_{\alpha}^{1} \rho$ по аналогии с подобной конструкцией в $D=4$, $N=2$ теории [10].

Суперполе $\rho$ обладает преобразованием центрального заряда, индуцированным соответствуюшим преобразованием кирального суперполя (5.11).

$N=2$ действие (5.6) можно переписать через обе $N=1$ компоненты $\widehat{\Phi}$ :

$$
\begin{aligned}
& -\frac{1}{2} \bar{C} \int d^{3} x(D)^{2} \phi+\text { c. c. }=\frac{1}{2} \int d^{3} x d^{2} \theta_{1}[(C-\bar{C}) \Sigma+i(C+\bar{C}) \rho]+\text { const } \\
& -\frac{1}{2} \int d^{7} z \widehat{H}(\widehat{\Phi})=-\frac{1}{2} \int d^{3} x\left(D^{1} D^{1}\right)\left(D^{2} D^{2}\right) \widehat{H}(\widehat{\Phi})= \\
& \quad=\frac{1}{2} \int d^{3} x d^{2} \theta_{1}\left\{\left[2 i \xi+\left(D^{1} D^{1}\right) \Sigma\right] \widehat{H}^{\prime}(\Sigma)+\frac{1}{2} \hat{\tau}(\Sigma) D^{1 \alpha} \rho D_{\alpha}^{1} \rho\right\}
\end{aligned}
$$

Отметим, что эти интегралы, включая линейный по $\rho$ член, инвариантны относительно преобразований $N=2$ суперсимметрии (6.14), (6.15).

Перейдем к анализу $N=1$ разложения калибровочного препотеншиала

$$
V_{s}\left(x, \theta_{1}, \theta_{2}\right)=\kappa\left(x, \theta_{1}\right)+i \theta_{2}^{\alpha} V_{\alpha}\left(x, \theta_{1}\right)+i\left(\theta_{2} \theta_{2}\right) M\left(x, \theta_{1}\right)
$$


и кирального калибровочного параметра

$$
\Lambda=\left[1+i \theta_{2}^{\alpha} D_{\alpha}^{1}+\left(\theta_{2} \theta_{2}\right)\left(D^{1} D^{1}\right)\right] \lambda\left(x, \theta_{1}\right) .
$$

Калибровочные преобразования $N=1$ компонент имеют вид

$$
\begin{aligned}
\delta_{\lambda} \kappa & =\lambda+\bar{\lambda} \\
\delta_{\lambda} V_{\alpha} & =D_{\alpha}^{1}(\lambda-\bar{\lambda}), \\
\delta_{\lambda} M & =-i\left(D^{1} D^{1}\right)(\lambda+\bar{\lambda}) .
\end{aligned}
$$

Таким образом, $\kappa$ - чисто калибровочная степень свободы, $V_{\alpha}-N=1$ калибровочное суперполе, $M$ - скалярная $N=1$ компонента $N=2$ супермультиплета.

Преобразования $N=2$ суперсимметрии для этих $N=1$ суперполей имеют следующий вид:

$$
\begin{aligned}
\delta_{2} \kappa & =-i \epsilon_{2}^{\alpha} V_{\alpha}, \\
\delta_{2} V_{\alpha} & =-\epsilon_{2 \alpha}\left[M+4 i|c|\left(\theta_{1} \theta_{1}\right)\right]-\frac{1}{2} \epsilon_{2}^{\beta} \partial_{\alpha \beta} \kappa, \\
\tilde{\delta}_{2} M & =\frac{1}{2} \epsilon_{2}^{\alpha} \partial_{\alpha \beta} V^{\beta} .
\end{aligned}
$$

Деформацию алгебры суперсимметрии (4.22) можно изучать в этом представлении.

Рассмотрим $N=1$ разложение линейного суперполя (4.17)

$$
\begin{gathered}
W_{s}\left(V_{s}\right)=\frac{i}{2}\left[\left(D^{1} D^{1}\right)+\left(D^{2} D^{2}\right)\right] V_{s}=w+i \theta_{2}^{\alpha} F_{\alpha}(V)-\left(\theta_{2} \theta_{2}\right)\left(D^{1} D^{1}\right) w \\
{\left[\left(D^{1} D^{1}\right)-\left(D^{2} D^{2}\right)\right] W_{s}=0, \quad\left(D^{1} D^{2}\right) W_{s}=0}
\end{gathered}
$$

где определены калибровочно-инвариантные скалярное и спинорное суперполя

$$
\begin{aligned}
w & =\frac{1}{2}\left[M+i\left(D^{1} D^{1}\right) \kappa\right], \\
F_{\alpha}(V) & =\frac{i}{2}\left(D^{1} D^{1}\right) V_{\alpha}+\frac{1}{4} \partial_{\alpha \beta} V^{\beta}, \quad D^{\alpha} F_{\alpha}=0 .
\end{aligned}
$$

Голдстоуновские преобразования $W_{s}(4.18)$ порождают следуюшие $\epsilon_{2}$-преобразования $N=1$ суперполей:

$$
\begin{aligned}
\delta w & =-i \epsilon_{2}^{\alpha} F_{\alpha} \\
\delta F_{\alpha} & =-\epsilon_{2 \alpha}\left[2|c|+i\left(D^{1} D^{1}\right) w\right]+\frac{i}{2} \epsilon_{2}^{\alpha} \partial_{\alpha \beta} w .
\end{aligned}
$$

Спинорная суперполевая напряженность $F_{\alpha}$ аналогична голдстоуновскому спинорному суперполю [11]. Оно описывает голдстоуновские степени свободы D2-браны, а суперполе $w$ соответствует массивным степеням свободы. Наша конструкция вводит $N=1$ калибровочное суперполе $V_{\alpha}$ как основной объект этой модели и позволяет изучать модификацию алгебры суперсимметрии на калибровочных полях D2-браны. Hетрудно переписать $N=2$ действие (4.8) через $N=1$ суперполя. 
Благодарности. Автор признателен Е. А. Иванову, С. О. Кривоносу, А. А. Капустникову, О. Лехтенфельду и особенно Е. Люкерскому за стимулирующие обсуждения и благодарит Е. Люкерского и Ж. Поповича за гостеприимство в университете Вроцлава, где была выполнена бо́льшая часть этой работы. Работа частично поддержана Российским фондом фундаментальных исследований (грант № 99-02-18417), фондом INTAS (INTAS-93-127-ext, INTAS-96-0308), программой Боголюбов-Инфельд и Узбекским фондом фундаментальных исследований (контракт № 11/27).

\section{Список литературы}

[1] J. Wess, J. Bagger. Supersymmetry and Supergravity. Princeton: Princeton University Press, 1983.

[2] P. West. Introduction to Supersymmetry and Supergravity. Singapore: World Scientific, 1986.

[3] S.J. Gates, M.T. Grisaru, M. Roček, W. Siegel. Superspace or One Thousand and One Lessons in Supersymmetry. Massachusetts: Benjamin Cummings, 1983.

[4] J. Hughes, J. Liu, J. Polchinski. Phys. Lett. B. 1986. V. 180. P. 370.

[5] A. Achucarro, J. Gauntlett, K. Itoh, P. K. Townsend. Nucl. Phys. B. 1989. V. 314. P. 129.

[6] Д. В. Волков, В. П. Акулов. Письма в ЖЭТФ. 1972. Т. 16. С. 621.

[7] J. Bagger, J. Wess. Phys. Lett. B. 1984. V. 138. P. 105.

[8] E. Ivanov, A. Kapustnikov. J. Phys. A. 1978. V. 11. P. 2375; Phys. Lett. B. 1990. V. 252. P. 439.

[9] J. Bagger, A. Galperin. Phys. Lett. B. 1994. V. 336. P. 25; Phys. Rev. D. 1997. V. 55. P. 1091.

[10] M. Roček, A. Tseytlin. Phys. Rev. D. 1999. V. 59. P. 106001.

[11] E. Ivanov, S. Krivonos. $N=1 D=4$ supermembrane in the coset approach. hep-th/9901003.

[12] I. Antoniadis, H. Partouche, T. R. Taylor. Phys. Lett. B. 1996. V. 372. P. 83.

[13] S. Ferrara, L. Girardello, M. Porrati. Phys. Lett. B. 1996. V. 376. P. 275.

[14] Е. А. Иванов, Б. М. Зупник. ЯФ. 1999. Т. 62. № 6. С. 1110; hep-th/9710236; E. A. Ivanov, B. M. Zupnik. Modifying $N=2$ supersymmetry via partial breaking. In: Theory of Elementary Particles. Proceedings of the 31th International Symposium on the Ahrenshop, 2-6 September 1997, Buckow, Germany. Eds. H. Dorn, D. Lüst, G. Weigt. Berlin: WILEY-VCH Verlag, 1998. P. 64-69; hep-th/9801016.

[15] R. Grimm, M. Sohnius, J. Wess. Nucl. Phys. B. 1978. V. 133. P. 275.

[16] E. A. Ivanov, S. O. Krivonos, A. I. Pashnev. Class. Q. Grav. 1991. V. 8. P. 19.

[17] W. Siegel. Nucl. Phys. B. 1979. V. 84. P. 193.

[18] B. M. Zupnik, D. G. Pak. Theor. Math. Phys. 1989. V. 77. P. 1070.

[19] O. Aharony, A. Hanany, K. Intriligator, N. Seiberg, M. J. Strassler. Nucl. Phys. B. 1997. V. 499. P. 67.

[20] J. de Boer, K. Hori, Y. Oz. Nucl. Phys. B. 1997. V. 500. P. 163.

Поступила в редакцию 8.VII.1999 г. 